\title{
A norbornyl route to cyclohexitols: stereoselective synthesis of conduritol-E, allo-inositol, MK 7607 and gabosines
}

\author{
Goverdhan Mehta* and Sripada Lakshminath \\ Department of Organic Chemistry, Indian Institute of Science, Bangalore 560 012, India
}

\begin{abstract}
A novel fragmentation sequence within the norbornane system, involving $C_{1}-C_{7}$ bond scission, provides convenient access to a highly functionalized and versatile cyclohexenoid building block which has been further elaborated to a range of cyclohexitols such as, conduritol E, allo-inositol and gabosine B. Our synthesis of the structure corresponding to gabosine $\mathrm{K}$ indicates that the structure of this natural product needs to be revised.
\end{abstract}

Keywords: fragmentation reactions; cyclitols; hydroxylation.

Molecular entities having cyclohexitol (polyhydroxylated cyclohexanoids) or amino cyclohexitol core structures have attracted a great deal of attention in recent years because of diverse biological activities exhibited by them, ranging from herbicidal and anti-microbial on the one hand to glycosidase inhibition and mediation of cellular communication on the other. ${ }^{1}$ Cyclohexitols with varied levels of oxygenation and stereochemical patterns are known and some typical examples are zeylenol $\mathbf{1}$, conduritol-E 2, allo-inositol 3, valienamine 4 and MK 7607 5. While several of the known cyclohexitols have been encountered in nature, many more have been accessed through syntheses to evaluate their biological potential. The syntheses of cyclohexitols remains an active area of current research and new approaches continue to be explored to achieve greater preparative efficiency and higher levels of stereo- and regiocontrol. ${ }^{1,2}$ In the accompanying communication, ${ }^{3}$ we have outlined a novel Groblike 'top-to-bottom' fragmentation sequence in a norbornyl derivative 6 to furnish a building block 7 for polyoxygenated cyclohexenoids and demonstrated its utility in the synthesis of carbasugars, e.g. fucopyranose 8, Scheme 1.<smiles>O[C@H]1C=C[C@@H](O)C(O)(O)[C@H]1O</smiles>

1<smiles>OC1C=C[C@@H](O)[C@H](O)[C@H]1O</smiles>

2<smiles>O[C@H]1[C@H](O)[C@H](O)[C@H](O)[C@@H](O)[C@H]1O</smiles>

3<smiles>N[C@H]1C=C(CO)[C@@H](O)[C@H](O)[C@H]1O</smiles>

4<smiles>OCC1=C[C@H](O)[C@H](O)[C@H](O)[C@H]1O</smiles>

5

\footnotetext{
* Corresponding author. E-mail: gm@orgchem.iisc.ernet.in (G. Mehta)
} 


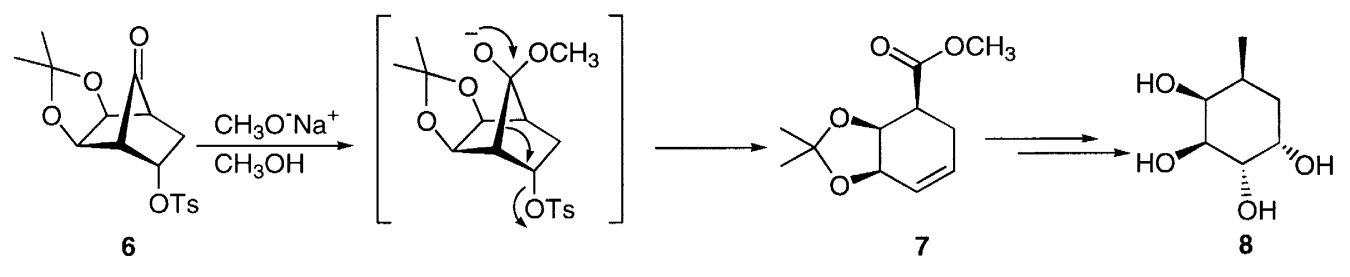

Scheme 1.

The structural similarity between $\mathbf{7}$ and the cis-dihydroxylated cyclohexenoids obtained from the microbial oxygenation ${ }^{2 b}$ of arenes spurred us to further explore the potential of 7 in a variety of cyclohexitol syntheses and these endeavours form the subject matter of this letter.

Our first objective was to elaborate $\mathbf{7}$ to conduritol and inositol type cyclohexitols. Towards this end, 7 was transformed to the bis-acetonide tosylate 9 and further elimination via the iodide gave the olefin $\mathbf{1 0}{ }^{6}$ Ozonolysis to cyclohexanone 11, reduction and mesylation furnished 12. ${ }^{6}$ Base mediated elimination to $\mathbf{1 3}^{6}$ and acetonide deprotection led to the conduritol-E 2, Scheme 2.4,6<smiles>[3H]C#CC#CC[Sb](C)(C)C</smiles>

Scheme 2. Reagents and conditions: (a) i. NaI, $\mathrm{Me}_{2} \mathrm{CO}, \Delta, 30 \mathrm{~h}, 92 \%$; ii. ${ }^{t} \mathrm{BuO}^{-} \mathrm{K}^{+},{ }^{t} \mathrm{BuOH}, \Delta, 20 \mathrm{~h}, 70 \%$; (b) $\mathrm{O}_{3}, \mathrm{NaHCO}_{3}$, $\mathrm{DCM},-78^{\circ} \mathrm{C}, 5 \mathrm{~min}, \mathrm{DMS}, 90 \%$; (c) i. $\mathrm{NaBH}_{4}, \mathrm{MeOH}, 0^{\circ} \mathrm{C}, 30 \mathrm{~min}, 89 \%$; ii. $\mathrm{MsCl}, \mathrm{Et}_{3} \mathrm{~N}, \mathrm{DCM},-10^{\circ} \mathrm{C}, 30 \mathrm{~min}, 90 \%$; (d) ${ }^{t} \mathrm{BuO}^{-} \mathrm{K}^{+}$, DMSO, rt, 2 h, 75\%; (e) Amberlyst-15, aq. $\mathrm{MeOH}, \mathrm{rt}, 20 \mathrm{~h}, 93 \%$

The bis-acetonide $\mathbf{1 3}$ also served as the precursor of allo-inositol 3. Catalytic osmium tetraoxide dihydroxylation of $\mathbf{1 3}$ was understandably slow but did lead to $14^{6}$ in reasonable yield. Deprotection in $\mathbf{1 4}$ furnished allo-inositol $\mathbf{3}$, which was conveniently characterised as the hexa-acetate 15. Scheme $3.5,6$

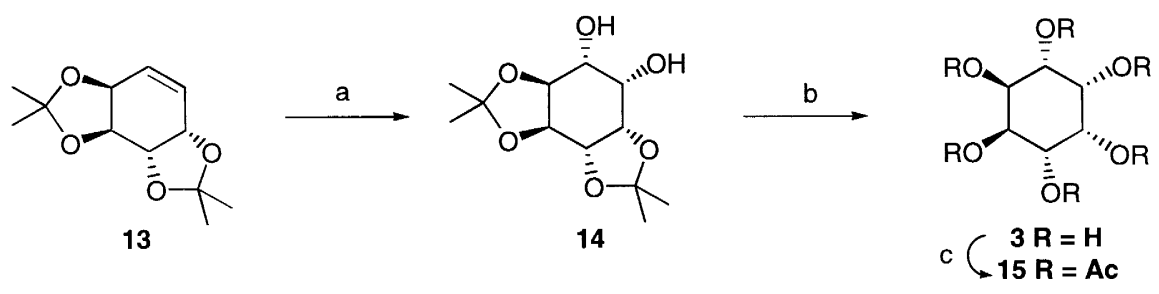

Scheme 3. Reagents and conditions: (a) $\mathrm{OsO}_{4}, \mathrm{NMMO}_{2} \mathrm{Me}_{2} \mathrm{CO}: \mathrm{H}_{2} \mathrm{O}$ (4:1), rt, 7 days, 70\%; (b) 5\% $\mathrm{HCl}, \mathrm{H}_{2} \mathrm{O}: \mathrm{Et}_{2} \mathrm{O}$ (4:1), $\mathrm{rt}$, $30 \mathrm{~h}$; (c) $\mathrm{Ac}_{2} \mathrm{O}, \mathrm{Py}, 80^{\circ} \mathrm{C}, 6 \mathrm{~h}, 65 \%$ (two steps)

Recently, structure elucidation of nearly a dozen novel secondary metabolites, named gabosines A-K, bearing the $\mathrm{C}_{7}$ skeleton of carbasugars has been reported from the various strains of Streptomycetes. ${ }^{7} \mathrm{We}$ recognised that our building block $\mathbf{7}$ and particularly the olefin $\mathbf{1 0}$ derived from it were well suited for the synthesis of gabosines. We first chose gabosine B $\mathbf{1 7}$ as the target and its synthesis was achieved in 
a straightforward manner. Exposure of $\mathbf{1 0}$ to rhodium trichloride resulted in isomerisation of the double bond to the desired tetra-substituted position and simple hydrolysis of $\mathbf{1 6}^{6}$ furnished gabosine B $\mathbf{1 7}$, identical in all respects with the natural product, Scheme $4 .{ }^{6}$ Stereoselective protonation in $\mathbf{1 6}$ during hydrolysis to furnish $\mathbf{1 7}$ as a single diastereomer is notable.<smiles>C=C1C[C@H]2OC(C)(C)O[C@H]2C2OC(C)(C)O[C@H]12</smiles>

10<smiles>CC1=C2OC(C)(C)O[C@H]2[C@H]2OC(C)(C)O[C@H]2C1</smiles>

16

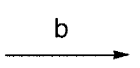<smiles>C[C@H]1C[C@@H](O)[C@H](O)[C@H](O)C1=O</smiles>

17

Scheme 4. Reagents and conditions: (a) $\mathrm{RhCl}_{3}, \mathrm{NaHCO}_{3}$, EtOH, $\Delta, 20 \mathrm{~h}, 60 \%$; (b) 5\% $\mathrm{HCl}, \mathrm{H}_{2} \mathrm{O}: \mathrm{Et}_{2} \mathrm{O}$ (4:1), $\mathrm{rt}, 90 \%$

We next turned our attention to the more embellished gabosine K 18 and the patented herbicide MK 76075 from Curvularia sp., ${ }^{8}$ closely related to the former. $\mathrm{OsO}_{4}$-mediated dihydroxylation of $\mathbf{1 0}$ led to readily separable diastereomeric diols 19 and 20 (70:30) (Scheme 5). ${ }^{6}$ Selective acetylation of the primary hydroxyl group in the major isomer 19 and dehydration furnished the olefins 21 and 22 (1:2) in which the latter could be recycled. ${ }^{6}$ A one-pot acetonide deprotection and acetate hydrolysis in 21 led to MK 7607. ${ }^{6}$ On the other hand, only acetonide deprotection in 21, under controlled conditions, resulted in $18,{ }^{9}$ the structure assigned to gabosine $\mathrm{K}$. However, we found that the spectroscopic data $\left({ }^{1} \mathrm{H}\right.$ and ${ }^{13} \mathrm{C}$ NMR) reported for gabosine $\mathrm{K}$ were different from our synthetic $\mathbf{1 8}$ and a direct comparison of the spectra provided by the authors confirmed this surmise. The structure of gabosine $\mathrm{K}$ therefore needs to be revised. ${ }^{9}$<smiles>C=C1C[C@H]2OC(C)(C)O[C@@H]2[C@H]2OC(C)(C)O[C@H]12</smiles>

$5 \mathrm{R}=\mathbf{H}$

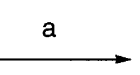<smiles>CC1(C)O[C@@H]2[C@H](O1)[C@@](O)(CO)C[C@@H]1OC(C)(C)O[C@H]12</smiles>

$\mathrm{b}$

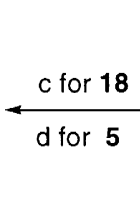<smiles>CC(=O)OCC1=C[C@@H]2OC(C)(C)O[C@H]2C2OC(C)(C)OC12</smiles>

21<smiles>CC1(C)OC2[C@H](O1)[C@](O)(CO)C[C@H]1OC(C)(C)O[C@@H]21</smiles>

20<smiles>CC(=O)O/C=C1\C[C@@H]2OC(C)(C)O[C@@H]2C2OC(C)(C)OC12</smiles>

22

$18 \mathrm{R}=\mathrm{Ac}$ (Previously assigned as gabosine $K$ )

Scheme 5. Reagents and conditions: (a) $\mathrm{OsO}_{4}, \mathrm{NMMO}, \mathrm{Me}_{2} \mathrm{CO}: \mathrm{H}_{2} \mathrm{O}(4: 1)$, rt, 2 days, 95\%; (b) i. $\mathrm{Ac}_{2} \mathrm{O}, \mathrm{DMAP}, \mathrm{DCM}, 0^{\circ} \mathrm{C}$, 30 min, 100\%; ii. $\mathrm{SOCl}_{2}$, Py, DCM, rt, 6 h, 45\% for 21 and 22; (c) Amberlyst-15, THF: $\mathrm{H}_{2} \mathrm{O}$ (2:3), rt, 48 h, 85\%; (d) 5\% $\mathrm{HCl}$, $\mathrm{H}_{2} \mathrm{O}: \mathrm{Et}_{2} \mathrm{O}(4: 1), \mathrm{rt}, 2$ days, $>95 \%$

In short, we have amplified the utility of the cyclohexenoid building block 7, obtained via a novel 'top-to-bottom' Grob-like fragmentation, in diverse cyclohexitol syntheses. Besides conduritol E 2 and allo-inositol 3, synthesis of these members of the gabosine family has been accomplished for the first time. 


\section{Acknowledgements}

We are grateful to Professor A. Zeeck for the copies of the spectra and helpful correspondence. S.L. thanks CSIR for the award of a Research Fellowship. Part of this work was initiated at the University of Hyderabad. We thank SIF at I.I.Sc. for high field NMR data.

\section{References}

1. Reviews: (a) Heightman, T. D.; Vasella, A. T. Angew. Chem., Int. Ed. Engl. 1999, 38, 750. (b) Suami, T.; Ogawa, S. Adv. Carbohydr. Chem. Biochem. 1990, 48, 21. (c) Suami, T. Top. Curr. Chem. 1990, 154, 257. (d) Berridge, M. J.; Irvine, R. F. Nature 1989, 341, 197.

2. (a) Balci, M.; Sutbeyaz, Y.; Secen, H. Tetrahedron 1990, 46, 3715. (b) Hudlicky, T.; Entwistle, D. A.; Pitzer, K. K.; Thorpe, A. J. Chem. Rev. 1996, 96, 1195 and references cited therein.

3. Mehta, G.; Mohal, N.; Lakshminath, S. Tetrahedron Lett. 2000, 41, 3505.

4. For some of the recent syntheses of Conduritol-E, see: (a) Landais, Y.; Angelaud, R. J. Org. Chem. 1996, 61, 5202. (b) Honzumi, M.; Hiroya, K.; Taniguchi, T.; Ogasawara, K. J. Chem. Soc., Chem. Commun. 1999, 1985. (c) Liu, P.; Dobbelaere, J.; Eycken, V.; Vandewalle, M. Synlett 1992, 243. (d) Hudlicky, T.; Luna, H.; Olivo, H. F.; Anderson, C.; Nugent, T.; Price, J. D. J. Chem. Soc., Perkin Trans. 1 1991, 2907.

5. (a) Motherwell, W. B.; Williams, A. S. Angew. Chem., Int. Ed. Engl. 1995, 34, 2031.(b) Tschamber, T.; Backenstrass, F.; Fritz, H.; Streith, J. Helv. Chim. Acta 1992, 75, 1052. (c) Mandel, M.; Hudlicky, T.; J. Chem. Soc., Perkin Trans. 1 1993, 741.

6. All new compounds reported here were racemic and characterized on the basis of spectroscopic data $\left({ }^{1} \mathrm{H}\right.$ and ${ }^{13} \mathrm{C} \mathrm{NMR}$, $J$ in $\mathrm{Hz})$ and elemental analyses. Selected spectroscopic data 10: $\delta_{\mathrm{H}}\left(300 \mathrm{MHz}, \mathrm{CDCl}_{3}\right): 5.19(1 \mathrm{H}, \mathrm{br} \mathrm{s}), 5.06(1 \mathrm{H}, \mathrm{br} \mathrm{s})$, $4.58(2 \mathrm{H}, \mathrm{ABq}, J 7), 4.47(2 \mathrm{H}, \mathrm{br} \mathrm{s}), 2.56(1 \mathrm{H}, \mathrm{br} \mathrm{d}, J 13), 2.16(1 \mathrm{H}, \mathrm{d}, J 13), 1.48(3 \mathrm{H}, \mathrm{s}), 1.37(6 \mathrm{H}, \mathrm{s}), 1.28(3 \mathrm{H}, \mathrm{s}) ; \delta_{\mathrm{C}}$ $\left(75 \mathrm{MHz}, \mathrm{CDCl}_{3}\right): 139.61,116.77,108.36,107.74,76.22,74.39,73.74,72.68,31.84,26.25,26.03,24.26,23.88 .13: \delta_{\mathrm{H}}$ $\left(300 \mathrm{MHz}, \mathrm{CDCl}_{3}\right): 5.73(2 \mathrm{H}, \mathrm{s}), 4.58-4.52(4 \mathrm{H}, \mathrm{m}), 1.38(6 \mathrm{H}, \mathrm{s}), 1.37(6 \mathrm{H}, \mathrm{s}) ; \delta_{\mathrm{C}}\left(75 \mathrm{MHz}, \mathrm{CDCl}_{3}\right): 126.93(2 \mathrm{C}), 109.14$ (2C), $73.24(2 \mathrm{C}), 70.23(2 \mathrm{C}), 27.83(2 \mathrm{C}), 26.43(2 \mathrm{C}) .2: \delta_{\mathrm{H}}\left(300 \mathrm{MHz}, \mathrm{D}_{2} \mathrm{O}\right.$, ref. acetone): $5.74(2 \mathrm{H}, \mathrm{m}), 4.17(2 \mathrm{H}, \mathrm{m})$, $3.78(2 \mathrm{H}, \mathrm{m}) ; \delta_{\mathrm{C}}\left(75 \mathrm{MHz}, \mathrm{D}_{2} \mathrm{O}\right.$, ref. acetone) $130.23(2 \mathrm{C}), 69.63(2 \mathrm{C}), 67.14(2 \mathrm{C}) .15: \delta_{\mathrm{H}}\left(300 \mathrm{MHz}, \mathrm{CDCl}_{3}\right): 5.52-5.42$ $(3 \mathrm{H}, \mathrm{m}), 5.36-5.3(3 \mathrm{H}, \mathrm{m}), 2.08(18 \mathrm{H}, \mathrm{s}) ; \delta_{\mathrm{C}}\left(100 \mathrm{MHz}, \mathrm{CDCl}_{3}\right): 169.71(3 \mathrm{C}), 169.45(3 \mathrm{C}), 67.60(3 \mathrm{C}), 67.21(3 \mathrm{C}), 20.71$ (3C), 20.64 (3C). 17: $\delta_{\mathrm{H}}\left(400 \mathrm{MHz}, \mathrm{CD}_{3} \mathrm{OD}\right): 4.41(1 \mathrm{H}, \mathrm{d}, J 10), 4.11-4.09(1 \mathrm{H}, \mathrm{m}), 3.45(1 \mathrm{H}, \mathrm{dd}, J 10,3), 2.97-2.87$ $(1 \mathrm{H}, \mathrm{m}), 2.11(1 \mathrm{H}$, ddd, $J 14,6,3.5), 1.42(1 \mathrm{H}, \mathrm{dt}, J 14,2), 1.03(3 \mathrm{H}, \mathrm{d}, J 6.5) ; \delta_{\mathrm{C}}\left(100 \mathrm{MHz}, \mathrm{CD}_{3} \mathrm{OD}\right): 211.52,78.26$, $78.19,69.78,38.18,37.88,13.75 .5: \delta_{\mathrm{H}}\left(400 \mathrm{MHz}, \mathrm{D}_{2} \mathrm{O}\right): 5.46(1 \mathrm{H}$, br s$), 4.12(1 \mathrm{H}$, br s), $4.05(1 \mathrm{H}$, br s), $3.95(2 \mathrm{H}$, br s $)$, $3.68(2 \mathrm{H}, \mathrm{br} \mathrm{s}) ; \delta_{\mathrm{C}}\left(100 \mathrm{MHz}, \mathrm{D}_{2} \mathrm{O}\right): 141.07,124.93,69.34,69.00,67.40,66.72,62.84$.

7. Bach, G.; Breiding-Mack, S.; Grabley, S.; Hammann, P.; Hutter, K.; Thiericke, R.; Uhr, H.; Wink, J.; Zeeck, A. Liebigs. Ann. Chem. 1993, 241.

8. Yoshikawa, N.; Chiba, N.; Mikawa, T.; Ueno, S.; Harimaya, K.; Iwata, M. Chem. Abstr. 1995, 122, 185533e.

9. Spectroscopic data for 18: $\delta_{\mathrm{H}}\left(400 \mathrm{MHz}, \mathrm{CD}_{3} \mathrm{OD}\right): 5.85-5.75(1 \mathrm{H}, \mathrm{m}), 4.70\left(1 \mathrm{H}, \frac{1}{2} \mathrm{ABq}, J 13.5\right), 4.60\left(1 \mathrm{H}, \frac{1}{2} \mathrm{ABq}, J\right.$ 13.5), 4.27-4.23 (2H, m), 3.90-3.87 (2H, m), $2.07(3 \mathrm{H}, \mathrm{s}) ; \delta_{\mathrm{C}}\left(75 \mathrm{MHz}, \mathrm{CD}_{3} \mathrm{OD}\right): 172.51,137.15,128.21,70.93,70.67$, 67.96, 67.40, 65.75, 20.78. Spectroscopic data reported for gabosine $\mathrm{K}: \delta_{\mathrm{H}}\left(200 \mathrm{MHz}, \mathrm{CD}_{3} \mathrm{OD}\right): 2.06\left(\mathrm{~s}, 9-\mathrm{H}_{3}\right), 3.39(\mathrm{~m}$, $2 \mathrm{H}, J 8,6.5,3-\mathrm{H}$ and $4-\mathrm{H}), 4.04-4.18(\mathrm{~m}, 2 \mathrm{H}, 1-\mathrm{H}$ and $2-\mathrm{H}), 4.53 / 4.73\left(2 \mathrm{~d}, J_{\mathrm{AB}} 13.5, \mathrm{~m}, 7-\mathrm{H}_{2}\right), 5.59(\mathrm{~m}, 6-\mathrm{H}) ; \delta_{\mathrm{C}}(50.3$ $\mathrm{MHz}, \mathrm{CD}_{3} \mathrm{OD}$ ): $172.5,136.2,128.8,77.5,77.1,73.5,73.0,64.8,20.8$. These spectral data reveal that the two compounds though not identical, do share common structural features indicating that the natural product may have a stereoisomeric structure related to $\mathbf{1 8}$. 